\title{
Body Focused Repetitive Behaviors (BFRBs) and Personality Features
}

\author{
Samuel R. Chamberlain • Brian L. Odlaug
}

Published online: 4 January 2014

(C) Springer International Publishing AG 2014

\begin{abstract}
Body focused repetitive behaviors (BFRBs) represent a collection of motoric acts that can become ingrained, habitual, and functionally impairing. They often relate to excessive grooming of the body or skin. Although these pathological habits have been described since ancient times, only recently have they been incorporated into psychiatric nosological systems. The relationship between BFRBs and aspects of personality has been scarcely researched. Understanding how formal axis-II personality disorders, questionnaire-based measures of personality, or other putatively enduring traits such as cognitive impairment, relate to the BFRBs may advance our understanding of the core characteristics of the BFRBs and subsequently lead to greater understanding of their pathophysiology and treatment. This article reviews the existing literature surrounding BFRBs and aspects of personality, and highlights limitations in our current understanding of these aspects, along with future research directions.
\end{abstract}

Samuel R. Chamberlain and Brian L. Odlaug denotes co-first author

Declaration of Interest Mr. Odlaug consults for Lundbeck Pharmaceuticals and received a grant from the Trichotillomania Learning Center. Mr. Odlaug has received honoraria and royalties from Oxford University Press. Dr. Chamberlain serves as a consultant for Cambridge Cognition

S. R. Chamberlain $(\square)$

Department of Psychiatry \& MRC/Wellcome Trust Behavioural and Clinical Neurosciences Institute, University of Cambridge,

Cambridge, United Kingdom

e-mail: srchamb@gmail.com

S. R. Chamberlain

Cambridge and Peterborough NHS Foundation Trust (CPFT),

Cambridge, United Kingdom

\section{B. L. Odlaug}

Department of Public Health, Faculty of Health and Medical

Sciences, University of Copenhagen, Øster Farimagsgade 5A, DK-1353 Copenhagen K, Denmark

e-mail: brod@sund.ku.dk
Keywords Body focused repetitive behavior · Cognition · Compulsivity $\cdot$ Dimension · Impulsivity $\cdot$ Personality · Self-regulation $\cdot$ Skin-picking $\cdot$ Trichotillomania

\section{Introduction}

The term 'body focused repetitive behaviors' (BFRBs) refers to a collection of motoric acts that are difficult to suppress, and which can become habitual and thereby functionally impairing e.g. $[1,2,3 \bullet]$. Some of these pathological behaviors have been recognized since ancient times: in work attributed to Hippocrates, it was recommended that physicians should examine whether patients pluck their hair, scratch, or weep as part of the general medical examination [4]. Nonetheless, the field of psychiatry was relatively late in formally recognizing BFRBs, beginning with the inclusion of trichotillomania in the Diagnostic and Statistical Manual Version III-Revised (DSM-III-R) [5].

BFRBs now represent core symptoms across several conditions in the Diagnostic and Statistical Manual, 5th Edition (DSM-5) [6]. Pathological hair-pulling (trichotillomania) and excoriation (skin-picking) disorder are explicitly recognized in the DSM-5, and fall under the category of 'Obsessive-Compulsive and Related Disorders'. Other types of habits are not listed as explicit separate disorders, but can warrant the broader diagnosis of BFRB disorder (an entity incorporated under 'Other Unspecified Obsessive-Compulsive and Related Disorders'). Examples of habits that can warrant a diagnosis of BFRB disorder include onychophagia (nail-biting), lip-biting, and cheek-biting. While not considered further for the purposes of this review, BFRB-like symptoms also occur in Stereotypic Movement Disorder (SMD), a neurodevelopmental disorder of childhood onset, most commonly reported in children with developmental disability, fetal alcohol exposure, and/or Lesch-Nyhan Syndrome. 
BFRBs have seldom been included in nationwide epidemiological screening studies, but multiple smaller studies suggest that - rather than being rare as believed by some trichotillomania, excoriation disorder, and BFRB disorders are quite common with estimated lifetime prevalence rates of $0.5-4 \%$ per disorder (in some cases, greater) [7-14, $15 \bullet \cdot, 16,17]$. For example, in a study conducted in 439 undergraduate psychology students at an urban American university, at least one BFRB (criteria modeled on DSM-IV) was identified in $13.7 \%$ of the sample, with nail-biting being the most common manifestation [18]. BFRB disorders are associated with marked functional impairment across several domains including in terms of school, work, social relationships, sports, and family life $[1,19 \bullet \bullet, 20,21 \bullet \bullet]$.

Unfortunately, and despite the relatively high prevalence and often chronically impairing and treatment resistant nature of these conditions, BFRB disorders have been quite neglected from a research point of view. This is the case both in terms of a lack of large scale epidemiological studies, but also a paucity of neurobiological investigations (few genetic, imaging, and cognitive studies).

Relationships between BFRBs and aspects of personality, whether one considers questionnaire-based measures on a spectrum (e.g. levels of impulsivity), formal axis-II personality disorders, or other putatively enduring traits such as cognitive impairment, are also poorly characterized. Such knowledge would be invaluable since personality aspects may influence the development of BFRB disorders, their persistence, and selection of appropriate treatments to maximize therapeutic benefit. This primer selectively reviews what is known of BFRBs and personality aspects.

\section{BFRBs and Personality: Categorical Versus Dimensional Approaches}

Personality disorders comprise ingrained and long-standing patterns of thoughts and/or behaviors that deviate from cultural expectations and are impairing. In the DSM-5, while the categorical approach towards personality disorders was retained, an alternative conceptualization was also included whereby personality was considered from the perspective of traits (dimensional rather than categorical approach) [6]. This decision was based, in part, on the suggestion that dimensional aspects of personality can contribute to the risk for, and persistence of, axis-I psychiatric disorders - perhaps to the same (or similar) degree as fully diagnosed personality disorders.

\section{BFRBs and Personality Disorders}

The extent to which BFRB disorders share comorbid overlap with personality disorders is under-explored, and evidence must be gleaned from relatively small studies conducted in samples that may not always have been representative of the general population.

\section{Trichotillomania}

The vast majority of available data derives from trichotillomania research which itself is extremely limited. Most of the available research has examined personality disorders in trichotillomania patients versus those with obsessive compulsive disorder (OCD), largely due to the characteristic overlap between the two disorders. One of the earliest studies to address this issue examined occurrence of personality disorders in eight patients with trichotillomania, identifying one or more personality disorders in two of the subjects [22]. In the same study, a numerically greater occurrence of one or more personality disorders was found in 13 patients with OCD (albeit this did not obtain statistical significance, likely reflecting the small sample size). In a study comprising 20 patients with trichotillomania and 20 patients with OCD, no statistically significant differences in rates of axis-II pathology were found between the two groups (precise rates were not reported) [23]. In a sample of 15 trichotillomania patients and 25 OCD patients, one or more personality disorders were found in three $(15 \%)$ of the former and $16(64 \%)$ of the latter; the rate was significantly higher in OCD than in trichotillomania [24]. In patients recruited from a variety of sources, individuals with trichotillomania $(n=49)$ versus OCD $(n=$ 130) manifested significantly lower lifetime rates of obsessive-compulsive personality disorder (OCPD; $13.3 \%$ versus $39.2 \%$ ) [25]. While not statistically significant, lifetime rates of other personality disorders were also numerically lower in trichotillomania than in OCD: namely, avoidant personality disorder $(0.03 \%$ versus $21.2 \%)$, schizotypal personality disorder ( $0 \%$ versus $5.3 \%$ ), and borderline personality disorder ( $8 \%$ versus $22.3 \%$ ). Studies comparing prevalence of personality disorders in trichotillomania against that in healthy controls are lacking, but one study compared 48 outpatients with trichotillomania to 48 psychiatric outpatient controls [26]. One or more personality disorders were found in $42 \%$ of trichotillomania subjects, a rate akin to the non-trichotillomania patient controls. Interestingly, there was some evidence for lower occurrence of borderline personality disorder in the subjects with trichotillomania compared to the patient controls.

Overall, these results suggest that trichotillomania appears to be associated with similar or lower occurrence of personality disorders when compared to certain other psychiatric conditions, especially OCD. Nonetheless, given that prevalence of personality disorders in the general population has been estimated at $5-10 \%[27,28]$, trichotillomania and personality disorders seem to co-occur more often than would be expected by chance alone. 


\section{Excoriation (Skin-Picking) Disorder}

To the authors' knowledge, only two studies have explored the occurrence of personality disorders in excoriation (skinpicking) disorder. In the first of such studies, conducted in 31 subjects with repetitive skin-picking and lacking a comparator group, the most common personality disorders were found to be obsessive-compulsive personality disorder (OCPD, $48 \%$ ) and borderline personality disorder (26\%) [29]. In the second study, rates of personality disorders were compared between 68 patients with trichotillomania, and 21 patients with excoriation disorder $[30 \bullet]$. There was a non-significant trend $(p=$ $0.06)$ towards higher occurrence of avoidant personality disorder in trichotillomania versus skin-picking (26.3\% versus $4.8 \%$ ). The occurrence rate of 'one or more' personality disorders was not reported. It is difficult to draw firm conclusions regarding relationships between skin-picking disorder and personality disorders based on these limited data.

\section{BFRB Disorder}

Since BFRB disorder is a relatively new diagnostic conceptualization, it is difficult to comment on likely overlap with personality disorders, since this issue has not yet been formally investigated. One would predict similar findings to those seen in trichotillomania and excoriation (skin-picking) disorder, given the considerable phenomenological overlap between these types of conditions [1]. In an early study, it was found that the occurrence of nail-biting in 62 people with sociopathy was significantly higher then was observed in 62 controls (48\% versus $24 \%$ ) [31].

\section{BFRBs and Personality Traits}

The assessment of personality traits is an evolving field, which traditionally focused on questionnaire-based approaches. Studies in the field of BFRBs have been somewhat varied not only in their choice of assessment instruments for personality traits, but also in the nature of subject enrollment criteria (for example, in the inclusion of people with repetitive hairpulling as opposed to a formal diagnosis; for example, in the source and methods of recruitment).

\section{Trichotillomania}

In a sample of 48 people with trichotillomania, personality traits (Minnesota Multiphasic Personality Inventory) were compared to those of 48 matched psychiatric patient controls. There was significantly less occurrence of cluster A personality traits, and better psychological adjustment on the MMPI in trichotillomania versus non-trichotillomania patient controls [26]. This result is somewhat akin to the data discussed previously which were suggestive of similar or lower rates of personality disorders in trichotillomania as compared to other psychiatric conditions in general. However, in a sample of internal medicine outpatients, individuals reporting a history of hair-pulling as an adult ( $2.9 \%$ of the sample) scored significantly higher on the borderline personality scale from the Personality Diagnostic Questionnaire (PDQ-4) [32], and on the Self-Harm Inventory (SHI), compared to other clinic attendees [33]. Some caution is warranted when interpreting these latter findings due to the self-report nature of the study, lack of objective trichotillomania diagnosis, and likelihood that personality disorders are disproportionately common in medical attendees. In a non-clinical sample of college students, hair-pullers $(n=44)$ showed significantly higher neuroticism scores than non hair-pullers $(n=244)$ on the Eysenck Personality Inventory (EPI) [34], but did not differ significantly in terms of extraversion or lie scores [35].

Turning to studies that have compared other aspects of personality in trichotillomania to OCD, one found that eight individuals with trichotillomania manifested significantly higher extraversion scores than 13 people with OCD [22], along with a trend towards higher neuroticism scores, as indexed by the Eysenck Personality Questionnaire (EPQ) [36]. Using the Temperament and Character Inventory (TCI) [37], a different study reported that 21 patients with trichotillomania scored higher than 68 patients with OCD on novelty seeking, but lower on harm avoidance [25]. Another study compared non clinical hair pullers $(n=66)$, trichotillomania $(n=18)$, and OCD $(n=29)$ on the EPQ or an equivalent index [38]. Subjects with non-clinical hairpulling and trichotillomania showed significantly higher extraversion scores than OCD patients, while the neuroticism and lie scales did not differ significantly across the three groups. Elsewhere, 68 people with trichotillomania showed high scores (as compared to normative data) on personality measures of reward dependence and harm avoidance, but normal scores on noveltyseeking [30••], as measured using the Tridimensional Personality Questionnaire (TPQ) [39]. Cumulatively, these results indicate that trichotillomania seems to be associated with greater extraversion than that seen in controls and in people with OCD, and that the findings with respect to novelty seeking and harm avoidance are inconsistent.

One study contrasted multiple personality features in trichotillomania not only with those seen in OCD, but also against borderline personality disorder [40]. The sample size in each group was 12, 17, and 14 subjects respectively. Trichotillomania and borderline personality disorder were both associated with lower obsessionality than OCD (interference subscale of the Leyton Obsessional Inventory), and with higher impulsivity than OCD (Impulsiveness on the Eysenck Impulsivity Questionnaire). Trichotillomania patients did not differ from the other groups in terms of aggression (Brown-Goodwin Lifetime Aggression History and Buss-Durkee Hostility Inventory), and TPQ measures. 


\section{Excoriation (Skin-Picking) Disorder}

Again, studies of other BRFBs besides trichotillomania are lacking. One study found that 21 patients with excoriation disorder scored highly (versus normative data) on reward dependence and harm avoidance, but normal on noveltyseeking [30••], as measured using the Tridimensional Personality Questionnaire (TPQ); this was similar to the findings for trichotillomania.

\section{BFRB Disorder}

As noted previously, BFRB disorder is a relatively new diagnostic entity; therefore, studies are lacking. There are some very early studies that explored aspects of personality traits in individuals with nail-biting, but these did not usually use structured questionnaires to quantify personality traits. One interesting study in 223 military personnel evacuated to a hospital due to nervous conditions reported high rates of nail-biting (occurring in $45 \%$ of subjects); note that subjects with psychosis or psychopathic personality types were excluded from this study. The authors reported that nail-biters tended to show elevated rates of irritability [41].

\section{Limitations of Traditional Personality Trait Measures: Could Cognitive Dysfunctions Represent Enduring Traits?}

While questionnaire-based measures relating to personality have proven useful in exploring aspects of BFRBs, it can be difficult to relate them to underlying brain function, as they cannot be adapted into translational models through which the neural and neurochemical substrates can be studied across animal species. Psychiatry has shifted towards a more neurobiologically grounded approach towards understanding different disorders, the overlap between them, and the mechanisms through which treatments exert their beneficial effects on symptoms. In this regard, cognitive tests might be useful as they can act as 'intermediate' markers that may indicate risk of developing BFRBs $[42,43]$. For example, in Gambling Disorder (see article elsewhere in this issue), certain cognitive problems have been identified not only in people with the full disorder, but also in people with subsyndromal symptoms at heightened risk for future development of the pathological manifestation of the condition. Initial work has explored various cognitive functions in people with BFRBs, mostly focusing on trichotillomania and, to a lesser extent, excoriation disorder. Deficits have been reported on various cognitive tests $[2,44 \bullet \cdot]$, particularly those quantifying aspects of impulse control and working memory.

It is not yet known whether cognitive deficits in people with BFRBs are 'state' (i.e. present with the illness and related to severity of illness) or 'trait' (occurring not only in patients, but also clinically unaffected first-degree relatives at heightened genetic risk of developing one or more BFRB disorder). This is an area of ongoing research. If cognitive measures could be shown to be trait in nature in BFRBs, this would support their use as enduring markers, and thereby as potential proxy 'personality'-related markers.

\section{Conclusions}

It is evident from this review that research into BFRBs and their relationship with personality is in its infancy. There is some evidence that personality disorders in trichotillomania are no more common than for other disorders, and may indeed be less common - for example, in comparison to OCD. Contrary to the view that hair-pulling in trichotillomania should be conceptualized as a type of self-injurious or selfharming behavior, borderline personality disorder appeared to occur less in trichotillomania than for some other conditions. One study found relatively high rates of OCPD and borderline personality disorder in excoriation disorder, but not in trichotillomania although differentiating between self-injurious picking versus picking in excoriation disorder is important both diagnostically and clinically [31]. This is interesting since it raises the prospect of potential differences between these two conditions that have traditionally been regarded as 'two sides of the same coin'. In general, the available personality disorder studies were relatively small and often lacked appropriate comparator groups.

Questionnaire-based measures of personality in BFRBs have also yield some insights, but, again, data were drawn from relatively few studies that often lacked robust comparator control groups. Trichotillomania was associated with higher rates of extraversion than $\mathrm{OCD}$, and with high scores compared to normative data on measures of reward-dependence and harmavoidance (while another study reported lower harm-avoidance in trichotillomania versus OCD). Cognitive based markers, if they can be shown to be 'trait' in nature and enduring, may in future offer a useful way of quantifying aspects of personality that can be more readily related to underlying brain dysfunction than can questionnaire-based measures.

We highlight the need for larger scale studies, with more rigorous designs, to better address these issues. Ideally future studies should incorporate comprehensive clinical screening for axis-II disorders, questionnaire-based personality measures, and salient neurocognitive tests, to explore the relationship between them. It is not yet known whether the cooccurrence of personality features in BRFBs has knock-on implications in terms of prognosis, type of treatment that the patient is likely to respond to, and neurobiology. If these issues can be explored, there are likely to be important clinical and theoretical implications. 


\section{Compliance with Ethics Guidelines}

Conflict of Interest Samuel Chamberlain serves as a consultant for Cambridge Cognition. Brian Odlaug serves as a consultant for Lundbeck Pharmaceuticals and received a grant from Trichotillomania Learning Center, and honoraria and royalties from Oxford University Press.

Human and Animal Rights and Informed Consent This article does not contain any studies with human or animal subjects performed by the author.

\section{References}

Papers of particular interest, published recently, have been highlighted as:

- Of importance and

-• Of major importance

1. Stein DJ et al. Is trichotillomania a stereotypic movement disorder? An analysis of body-focused repetitive behaviors in people with hair-pulling. Ann Clin Psychiatry. 2008;20(4):194-8.

2. Stein DJ, Chamberlain SR, Fineberg N. An A-B-C model of habit disorders: hair-pulling, skin-picking, and other stereotypic conditions. CNS Spectrosc. 2006;11(11):824-7.

3. Snorrason I et al. Skin picking disorder is associated with other body-focused repetitive behaviors: findings from an internet study. Ann Clin Psychiatry. 2012;24(4):292-9. Insightful study using an internet based approach to explore the relationship between several types of body focused repetitive behaviors.

4. Chadwick J and Mann WN. Hippocratic writings, translated by authors. 1983: Penguin books.

5. DSM-III-R. 1987: American Psychiatric Association.

6. Association, A.P. Diagnostic and statistical manual of mental disorders (5th ed.) (DSM-5). Arlington: American Psychiatric Publishing; 2013.

7. Christenson GA, Mansueto CS. Trichotillomania: descriptive statistics and phenomenology. In: Stein DJ, Christenson GA, Hollander E, editors. Trichotillomania. Washington: Am Psych; 1999. p. 1-41.

8. Dell'Osso B et al. Epidemiologic and clinical updates on impulse control disorders: a critical review. Eur Arch Psychiatry Clin Neurosci. 2006;256(8):464-75.

9. Duke DC, et al. The phenomenology of hairpulling in a community sample. J Anxiety Disord. 2009;23(8):1118-25.

10. Keuthen NJ et al. The prevalence of pathologic skin picking in US adults. Compr Psychiatry. 2010;51(2):183-6.

11. Mansueto CS, Thomas AM, Brice AL. Hair pulling and its affective correlates in an African-American university sample. J Anxiety Disord. 2007;21(4):590-9.

12. Al-Jawadi AA, Abdul-Rhman S. Prevalence of childhood and early adolescence mental disorders among children attending primary health care centers in Mosul, Iraq: a cross-sectional study. BMC Public Health. 2007;7:274.

13. Bohne A et al. Skin picking in German students. Prevalence, phenomenology, and associated characteristics. Behav Modif. 2002;26(3):320-39.

14. Odlaug BL and Grant JE. Impulse-control disorders in a college sample: results from the self-administered Minnesota Impulse Disorders Interview (MIDI). Prim Care Companion J Clin Psychiatry, 2010. doi:10.4088/PCC.09m00842whi.

15.• Odlaug BL, Grant JE. Pathologic skin picking. Am J Drug Alcohol Abuse. 2010;36(5):296-303. Detailed introduction to excoriation disorder for the interested reader.
16. Odlaug BL et al. Skin picking disorder in university students: health correlates and gender differences. Gen Hosp Psychiatry. 2013;35(2):168-73.

17. Siddiqui EU et al. Prevalence of body-focused repetitive behaviors in three large medical colleges of Karachi: a cross-sectional study. BMC Res Notes. 2012;5:614.

18. Teng EJ et al. Body-focused repetitive behavior problems. Prevalence in a nonreferred population and differences in perceived somatic activity. Behav Modif. 2002;26(3):340-60.

19.• Woods DW et al. The Trichotillomania Impact Project (TIP): exploring phenomenology, functional impairment, and treatment utilization. J Clin Psychiatry. 2006;67(12):1877-88. The Trichotillomania Impact Project (TIP) is a large scale collaboration providing new insights into BFRBs. The paper provides information on TIP and what has been found in relation to different aspects of the condition.

20. Diefenbach GJ et al. Trichotillomania: impact on psychosocial functioning and quality of life. Behav Res Ther. 2005;43(7):869 84.

21.• Tucker BT et al. The Skin Picking Impact Project: phenomenology, interference, and treatment utilization of pathological skin picking in a population-based sample. J Anxiety Disord. 2011;25(1):88-95. Similar type of large scale collaboration as for trichotillomania, this time applied to excoriation disorder.

22. Stanley MA et al. A comparison of clinical features in trichotillomania and obsessive-compulsive disorder. Behav Res Ther. 1992;30(1):39-44.

23. Himle JA, Bordnick PS, Thyer BA. A Comparison of Trichotillomania and Obsessive-Compulsive Disorder. J Psychopathol Behav Assess. 1995;17(3):251-60.

24. Tukel $\mathrm{R}$ et al. Comparison of clinical characteristics in trichotillomania and obsessive-compulsive disorder. J Anxiety Disord. 2001;15(5):433-41.

25. Lochner $\mathrm{C}$ et al. Obsessive-compulsive disorder and trichotillomania: a phenomenological comparison. BMC Psychiatry. 2005;5(1): 2.

26. Christenson GA, Chernoff-Clementz E, Clementz BA. Personality and clinical characteristics in patients with trichotillomania. J Clin Psychiatry. 1992;53(11):407-13.

27. Huang $\mathrm{Y}$ et al. DSM-IV personality disorders in the WHO World Mental Health Surveys. Br J Psychiatry. 2009;195(1):46-53.

28. Lenzenweger MF et al. DSM-IV personality disorders in the National Comorbidity Survey Replication. Biol Psychiatry. 2007;62(6):553-64.

29. Wilhelm $\mathrm{S}$ et al. Self-injurious skin picking: clinical characteristics and comorbidity. J Clin Psychiatry. 1999;60(7):454-9.

30.• Lochner $\mathrm{C}$ et al. Trichotillomania and skin-picking: a phenomenological comparison. Depress Anxiety. 2002;15(2):83-6. An important study exploring similarities and differences between these two conditions, which traditionally have been regarded by many to be 'one and the same'..

31. Walker BA, Ziskind E. Relationship of nailbiting to sociopathy. J Nerv Ment Dis. 1977;164(1):64-5.

32. Hyler SE. Personality Diagnostic Questionnaire-4. New York: New York State Psychiatric Institute; 1994.

33. Sansone RA, Wiederman MW, Sansone LA. The Self-Harm Inventory (SHI): development of a scale for identifying selfdestructive behaviors and borderline personality disorder. J Clin Psychol. 1998;54(7):973-83.

34. Eysenck HJ, Eysenck SBG. Manual for the Eysenck Personality Inventory. San Diego: Educational and Industrial Testing Service; 1968.

35. Stanley MA et al. Nonclinical Hair Pulling: Phenomenology and Related Psychopathology. J Anxiety Disord. 1994;8(2):119-30.

36. Eysenck HJ, Eysenck SBG. Manual for the Eysenck Personality Questionnaire. London: Hodder \& Stoughton; 1978. 
37. Cloninger CR et al. The Temperament and Character Inventory: A guide to its development and use. Missouri: Center for Psychobiology of Personality, Washington University, St. Louis; 1994.

38. Stanley MA et al. Nonclinical hair-pulling: affective correlates and comparison with clinical samples. Behav Res Ther. 1995;33(2): 179-86.

39. Cloninger CR, Przybeck TR, Svrakic DM. The Tridimensional Personality Questionnaire: U.S. normative data. Psychol Rep. 1991;69(3 Pt 1):1047-57.

40. Stein DJ et al. Compulsive and impulsive symptomatology in trichotillomania. Psychopathology. 1995;28(4):208-13.
41. Hill JM. Nail biting; incidence, allied personality traits and military significance. Am J Psychiatry. 1946;103(2):185-7.

42. Gottesman II, Gould TD. The endophenotype concept in psychiatry: etymology and strategic intentions. Am J Psychiatry. 2003;160(4):636-45.

43. Chamberlain SR, Menzies L. Endophenotypes of obsessivecompulsive disorder: rationale, evidence and future potential. Expert Rev Neurother. 2009;9(8):1133-46.

44.• Chamberlain SR et al. Trichotillomania: neurobiology and treatment. Neurosci Biobehav Rev. 2009;33(6):831-42. More detailed introduction to trichotillomania for the interested reader. 